POS PROCEEDINGS

\title{
Operation and performance of the ATLAS liquid argon electromagnetic calorimeters
}

\author{
Julien Maurer*† \\ Aix-Marseille University, CPPM, CNRS/IN2P3 \\ E-mail: jmaurer@cern.ch
}

\begin{abstract}
The electromagnetic calorimetry is a key component for the achievement of the ATLAS experiment physics goals. It must provide an excellent energy resolution in a vast domain $(1 \mathrm{GeV}$ to several $\mathrm{TeV}$ ) as well as great abilities for electron and photon identification. This document summarizes its performances reached in the first 3 years of data taking at the LHC.
\end{abstract}

The European Physical Society Conference on High Energy Physics -EPS-HEP2013

18-24 July 2013

Stockholm, Sweden

\footnotetext{
*Speaker.

${ }^{\dagger}$ on behalf of the ATLAS Liquid Argon Group
} 

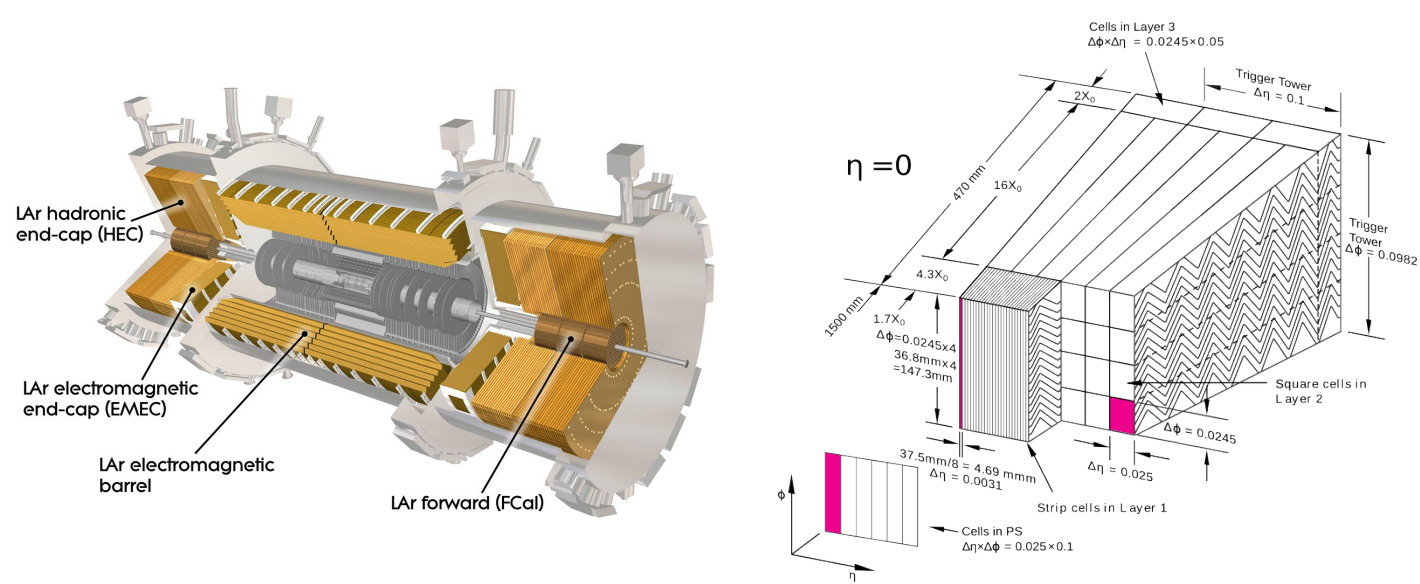

Figure 1: Overview of the calorimetry system, and segmentation of the EM calorimeter in the central region.

\section{The ATLAS electromagnetic calorimeters}

The ATLAS detector [1] employs liquid argon sampling calorimeters [2] for several parts: electromagnetic (EM barrel ${ }^{1} 0 \leq|\eta| \leq 1.5$, EM end-caps $1.4 \leq|\eta| \leq 3.2$ ), hadronic end-caps $(1.5 \leq|\eta| \leq 3.2)$ and forward $(3.1 \leq|\eta| \leq 4.9)$. The EM lead absorber plates are mounted in an accordion structure (Fig. 1) allowing a near-perfect uniformity of the response in $\varphi$. Ionization charges are collected by triple foil electrodes positioned in the middle of the gap between two absorbers, and held by a honeycomb structure filled with argon. High voltage (1 to $2 \mathrm{kV}$ ) is supplied on each electrode side by two independent sources, to limit the risk of dead areas. The electrodes are segmented in three longitudinal layers (Fig. 1) to improve energy reconstruction and electron/photon identification. The front layer is finely divided along $\eta$ for precise $\eta$ measurement and $\pi^{0}$ discrimination, the middle layer collects most of the shower energy, and the back layer allows to correct for shower energy leakage beyond the calorimeter. The region $|\eta|<1.8$ is equipped with a presampler built from planar electrodes and argon gaps, to detect early showers triggered when particles interact with the material in front of the calorimeter and improve energy linearity.

\section{Operation of the calorimeter}

Dedicated electronic calibration runs are taken daily during accelerator interfills, by injecting a known signal in the electrodes, and measuring each channel's pedestal, noise and gain. These parameters are very stable with time (pedestal drift $<3 \mathrm{MeV}$, gain $<0.03 \%$ ). The liquid argon temperature homogeneity and uniformity is monitored continuously by 508 probes, as its impact on the energy scale is about $-2 \% / \mathrm{K}$. The measured temperature excursions are of the order of $60 \mathrm{mK}$ for a tolerance threshold of $100 \mathrm{mK}$. Argon purity is also well under control, the level of impurities being $200 \mathrm{ppb}$ in the barrel and $140 \mathrm{ppb}$ in the end-caps while the accepted limit is $1000 \mathrm{ppb}$.

Issues in the liquid argon calorimeters have been responsible in 2012 for the loss of $0.88 \%$ of recorded data. The main contribution $(0.46 \%$ in 2012) originates from sporadic trips of the high voltage supply modules. It has been substantially reduced compared to earlier operation (1\% loss

\footnotetext{
${ }^{1}$ Here and following: indication of the coverage in pseudo-rapidity $\eta=-\ln [\tan (\theta / 2)]$
} 
in 2011) thanks notably to the installation of new modules able to tolerate short current overloads. Another source of loss is due to recurrent bursts of coherent noise affecting most of the channels of a subsystem (mostly the end-caps). Such events, only seen in presence of collisions, have a very short duration (typically $5 \mu \mathrm{s}$ ) but frequently occur at high luminosity (roughly one per minute). All data recorded in a short time window around these events is rejected. The 2012 data loss associated to this veto amounts to $0.2 \%$, much less than for 2011 operation in which $1.2 \%$ data were lost due to the lack of an efficient procedure to spot these noise bursts before data reconstruction.

The operational fraction of the liquid argon calorimeter is excellent: more than $99.9 \%$ channels are usable. A limited fraction of cells $(<0.5 \%)$ are also affected by a sporadic noise. This noise can be however well identifed by the use of the quality factor of the pulse shape, which quantifies the agreement with the reference shape measured during the calibration runs. Furthermore, these few cells being mostly localised in the presampler, the impact on the accuracy is negligible.

\section{Performances: energy measurement and particle identification}

Electrons and photons are built from fixed size rectangular clusters locally maximizing the contained energy, and classified according to the presence of a matching track. The cluster energy calibration accounts for losses in front or beyond the EM calorimeter, and lateral leakage due to the fixed cluster size, through a parametrization tuned with the simulation.

The energy resolution of the calorimeter can be parametrized as $\frac{\sigma_{E}}{E}=\frac{a}{\sqrt{E}} \oplus \frac{b}{E} \oplus c$ with a stochastic term $a \sim 10 \% \mathrm{GeV}^{1 / 2}$, a noise term $b$ negligible for the considered energies, and a constant term $c$ measured in test beams to be $0.7 \%$. The electron energy scale is determined in situ [3] with a $Z \rightarrow e^{+} e^{-}$selection; the amplitude of the scale corrections applied to data events are about $2 \%$ in the barrel and $4 \%$ in the end-caps. An excellent stability of this scale regarding time or pile-up has been observed, with a standard deviation of respectively $0.033 \%$ and $0.015 \%$. The effective constant term of the resolution has been determined from $Z \rightarrow e^{+} e^{-}$lineshape with 2010 data to be $1.2 \pm 0.6 \%$ in the barrel, and $1.8 \pm 0.6 \%$ in the end-caps [3]. An improved mapping of the material in front of the calorimeter may bring in the future these values closer to the aimed $0.7 \%$.

Electron and photon identifications take advantage of the high granularity of the calorimeter and rely on discriminant variables built from the shower shape (e.g. lateral width, leakage in the hadronic layer) as well as tracking information for electrons, in particular the transition radiation abilities of the tracker outermost component. A $90 \%$ electron identification efficiency is obtained [3] for a typical jet rejection factor of 5000, with good robustness to pile-up. Similar performances are reached for photon identification.

\section{References}

[1] ATLAS Collaboration, The ATLAS experiment at the CERN Large Hadron Collider, JINST 3 (2008) S08003

[2] ATLAS Collaboration, Readiness of the ATLAS liquid argon calorimeter for LHC collisions, Eur. Phys. Journ. C70 (2010) 723-753

[3] ATLAS Collaboration, Electron performance measurements with the ATLAS detector using the 2010 LHC proton-proton collision data, Eur. Phys. Journ. C72 (2011) 1-46 